\title{
Droplet spreading and wettability of abrasive processed aluminum alloy surfaces
}

\author{
G.V. Kuznetsov ${ }^{\text {a }}$, E.G. Orlova ${ }^{\text {a, }}$, D.V. Feoktistov ${ }^{\text {b }}$, A.G. Islamova ${ }^{\text {a }}$, A.V. Zhuikov ${ }^{c}$ \\ ${ }^{a}$ National Research Tomsk Polytechnic University, Lenin Ave., 30, 634050, Tomsk, Russia \\ ${ }^{\mathrm{b}}$ Institute of Thermophysics Siberian Branch, Russian Academy of Sciences, Lavrentiev Ave. 1, Novosibirsk, \\ 630090, Russia \\ ${ }^{c}$ Siberian Federal University, Svobodny Ave. 79, Krasnoyarsk, 660041, Russia
}

\begin{abstract}
.
One of the main cause of a droplet metastable state is found to be surface roughness. This state is characterized by a large contact angle hysteresis and condition when the static contact angle is larger than the advancing dynamic contact angle. Besides the texture, other factors can influence the deviation from the equilibrium state, in particular, the fluid flow rate (the growth rate of a droplet) affecting the contact line speed. An experimental study was done to determine the effect of roughness and fluid flow rate on wetting of aluminum-magnesium alloy surfaces with random roughness processed by abrasive polishing. Three-dimensional roughness parameters were used to evaluate their texture. The correlations between these parameters, static, advancing and receding dynamic contact angles, hysteresis, and contact line speed were obtained. The molecular-kinetic theory of wetting was used to interpret the dynamic contact angle data.
\end{abstract}

Keywords: roughness; abrasive processing; three-dimensional roughness parameters; wetting; dynamic contact angle; contact angle hysteresis.

\section{Introduction}

Wetting and spreading are ubiquitous in processing and re-processing of materials [1-4]: jet printing, spray cooling, surface coating, painting and lubrication. Enhancement of wetting properties can provide lubrication and help to reduce wear and friction. In mentioned and similar processes, the technical surfaces used are not molecularly smooth; their texture is characterized by a chaotic arrangement of nano- and micro-cavities and asperities. Despite the fact that in recent decades, methods for controlling wetting and spreading by creating nano- and microscaled textures (laser processing, lithography, etching, abrasive processing, and additive technologies) are being extensively developed, theoretical frameworks to predict changes in wettability and spreading have not yet been developed. To do it, it is necessary to obtain experimental data relating quantitative characteristics of the texture (three dimensional roughness parameters) with wetting properties and spreading process.

The quantitative wetting characteristic on the molecularly smooth surfaces is an equilibrium contact angle defined by the Young equation [5]. This angle is dependent on the interfacial tensions between solid and gas $\sigma_{\mathrm{sg}}$, solid and liquid $\sigma_{\mathrm{sl}}$, and liquid and gas $\sigma_{\mathrm{lg}}$. Engineering surfaces differ from molecularly smooth by roughness, the contact angle here deviates from the equilibrium by the value of the contact angle hysteresis (CAH) [6]. The last mentioned is defined

\footnotetext{
${ }^{*}$ Corresponding author.

E-mail address: $\underline{\text { lafleur@tpu.ru }}$
} 
as the difference between the advancing $\theta_{0 \mathrm{~A}}$ and receding $\theta_{\mathrm{OR}}$ dynamic contact angles (DCA) measured while the contact line (CL) pins as it moves towards the gas and liquid phases, respectively:

$$
\Delta \theta=\theta_{0 \mathrm{~A}}-\theta_{0 \mathrm{R}}
$$

where $\theta_{0 \mathrm{~A}}$ and $\theta_{0 \mathrm{R}}$ are the advancing and receding dynamic contact angles when the CL pins, respectively.

The static contact angle $\theta_{\mathrm{S}}$ formed by a droplet and surface is suggested to lie between $\theta_{0 \mathrm{~A}}$ and $\theta_{\text {OR }}$ [7].

The influence of the contact line speed $V$ on the DCAs and CAH while spreading of a droplet over rough metal surfaces has not been studied at the level of prognostic evaluation. However, it is known [6] that when the CL speeds do not exceed a few micrometers per second, the advancing and receding DCAs are close or equal to the static one. The advancing DCA on super-hydrophobic surfaces does not depend on the CL speed, and the receding DCA decreases when $V$ increases [7].

There are theories [8-11] to describe the dependency of the dynamic contact angles on the speed of the liquid contact line. One of them is the molecular-kinetic theory (MKT) $[8,9]$ based on the kinetics of molecular processes occurring on the three-phase contact line [8]. The energy dissipation in the vicinity of the contact line is taken into account, and it moves through individual molecular jumps, with an equilibrium frequency $k^{0}$ and a displacement distance $\lambda$ :

$$
V=2 k^{0} \lambda \sinh \left(\frac{\sigma \lambda^{2}\left(\cos \theta-\cos \theta_{d}\right)}{2 k_{B} T}\right)
$$

where $\theta_{d}$ is the dynamic contact angle, $\theta$ is the equilibrium contact angle; $\sigma$ is the surface tension; $k_{B}$ is the Boltzmann's constant, and $T$ is the temperature.

If the dynamic contact angles are close to the equilibrium, MKT equation (2) reduces to [9]:

$$
V=\frac{\sigma}{\xi}\left(\cos \theta-\cos \theta_{d}\right)
$$

where $\xi=k_{B} \cdot T / k^{0} \cdot \lambda^{3}$ is the friction coefficient at the contact line, $\mathrm{Pa} \cdot \mathrm{s}$.

Parameters $\lambda, k^{0}$ and $\xi$ in equations (2) and (3) are obtained from the experiment by curve fitting procedures, i.e. it is impossible to define their values before conducting an experiment. It was found that the MKT equation fitted satisfactorily the experimental dependencies of the DCA on the CL speed obtained for water and aqueous solutions spreading over polyethylene terephthalate surfaces (PET) [12]. However, its applicability for the case of spreading on rough metal surfaces has not been still established.

There are experimental works [13-16] aimed at studying spreading and wetting properties of molecularly smooth surfaces $[13,16]$ or surfaces with an order texture $[14,15,17]$. Fewer works are devoted to the study of such processes on engineering surfaces of metals with random roughness $[18,19]$. It is connected with the difficulties in quantitative assessment of the texture of surfaces with random roughness, in particular, when choosing roughness parameters from the existing standards [20]. Usually, one parameter of roughness was used: $\operatorname{Ra}[21,22]$ characterizing the arithmetic average of the roughness profile, or Rz [23], maximum height of the irregularities. In [19], parameters Rmr, the relative material ratio of the roughness profile, and Rlo, the developed length of the roughness profile, were used to modify the Wenzel [24] and Cassie [25] equations and obtain the static contact angle of tested aluminum surfaces prepared by 
abrasive polishing. However, all these parameters are two-dimensional, describe the texture along the profile and do not take into account the location of irregularities on the surface.

An increase in roughness, as it is known [21-23, 26], can both reduce [22, 23] and increase $[21,26]$ the static contact angle. The decrease in $\theta_{\mathrm{s}}$ and increase in the standard deviation of several repeated measurements were recorded in [22] with the growth of roughness on titanium, aluminum and copper surfaces processed by papers of grade 600\#, 1500\# and 2000\#. Obtained dependencies were explained by the state of a drop characterized by the Wenzel equation [24]. Exponential dependence of $\theta_{\mathrm{S}}$ on the sandpaper grain size used for processing of wood species was obtained in [23]. This is characterized by three regions: 1) decrease in the static contact angle due to an increase in adhesion; 2) reaching its minimum value; 3) a slight increase in $\theta_{\mathrm{S}}$. The growth of roughness of glassy carbon [26] processed by abrasive papers of grit size 1200, 800 , and 400 led to a decrease in wettability (static contact angle changed from $62.11^{\circ}$ to $92.32^{\circ}$ ). The latter does not correlate to the Wenzel model [24] (the increase in the roughness factor is accompanied by a decrease in the contact angle when the surface is hydrophilic). Such a contradictory wetting behavior was recorded for processed aluminum and stainless steel surfaces [21]. Moreover, the textures obtained by two different methods (abrasive polishing and etching) and characterized by the same value of Ra show different wetting angles [21]. In addition to roughness [27], $\theta_{\mathrm{s}}$ significantly depends on the state of a drop on the surface (Cassi-Baxter or Wenzel) and the method of its cleaning after abrasive processing.

Results of the present experimental studies contribute to the development of physical insights of wetting and droplet spreading over rough aluminum-magnesium alloy surfaces. This alloy is one of the most widely used metals for the manufacture of the technical surfaces. Threedimensional roughness parameters were used in this study for quantitative assessment of surfaces processed by abrasive polishing by discs with different grit size. The studies are aimed at establishing a correlation between the contact angle, hysteresis, and contact line speed with the quantitative texture characteristics (3D roughness parameters) under the conditions of varying fluid flow rate. The linear and classical equations of the molecular-kinetic theory of spreading were used to connect the dynamic contact angle with the contact line speed for the case of distilled water droplet spreading over rough aluminum-magnesium alloy surfaces.

\section{Materials and methods}

The shadow optical method was used to conduct the experimental studies of wetting and droplet spreading over metal surfaces (Fig. 1) [28-30]. 


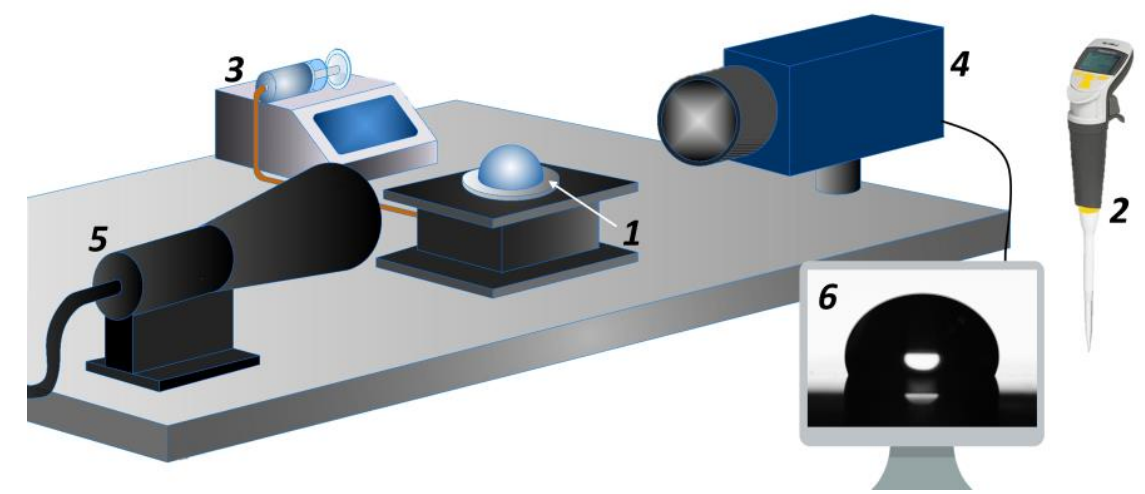

Fig. 1 Schematic representation of the experimental setup: 1 - substrate; 2 - single-channel electronic dispensing device; 3 - syringe pump; 4 - high-speed video camera; 5 - source of plane-parallel light; 6 - computer with shadow image of a droplet

Surface wetting was characterized by the static contact angle measured while placing a droplet of distilled deionized nano-filtered Milli-Q water $(10 \mu \mathrm{l})$ on substrate 1 by single-channel electronic dispensing device Lenpipet Stepper (produced by Thermo Scientific) 2. Contact radius ( $r$ ) of a droplet with a volume of $10 \mu \mathrm{l}$ on polished surface did not exceed $1.9 \mathrm{~mm}$, that was smaller the capillary constant of distilled water $a=3.8 \mathrm{~mm}$. In such a condition $(r \leq a$, where $a=\sigma / \rho g$, with $\rho$ is liquid density and $g$ is gravity acceleration) a droplet size did not affect the static contact angle.

The contact line dynamics was studied by forced pumping and withdrawing of liquid on a surface by system consisting of high-precision electronic syringe pump (produced by Cole Parmer) 3 and copper channel $1 \mathrm{~mm}$ in diameter connecting a pump with an $0.4 \mathrm{~mm}$ opening in the substrate center (Fig. 1). The liquid was supplied and pumped out through the opening on the underside of the substrate (Fig. 2). In the experiments on the contact line dynamics, the volume of a droplet $(30 \mu \mathrm{l})$ did not change. The liquid flow rate determining the contact line speed was varied by the pump in the range from 5 to $100 \mu \mathrm{l} / \mathrm{s}$. The flow rates were chosen according to the technical capabilities of the liquid dispensing system and methods for processing the shadow images of droplets. The contact line speed was defined according to change in the droplet diameter when pumping and withdrawing of liquid.

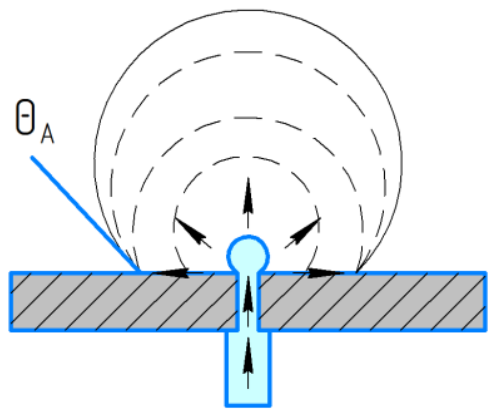

(a)

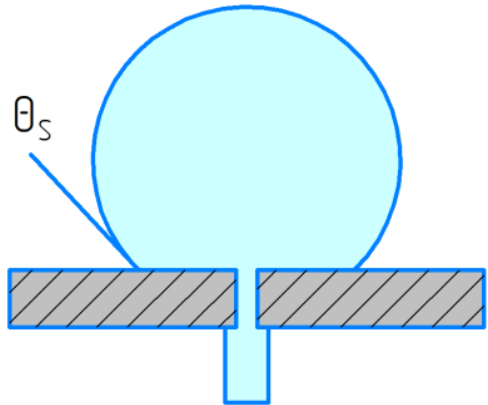

(b)

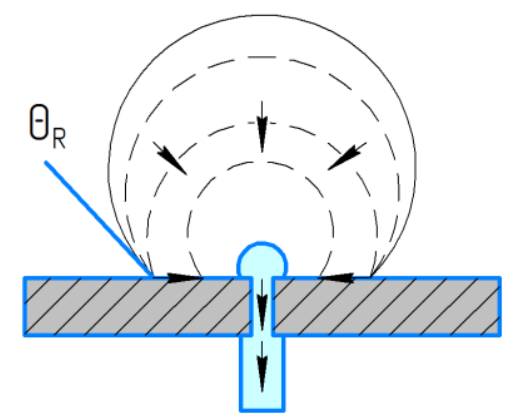

(c)

Fig. 2 Schematic representation of experiment on the contact line dynamics: (a) pumping of liquid (formation of the advancing dynamic contact angle $\theta_{\mathrm{A}}$ ); (b) static contact angle $\left(\theta_{\mathrm{S}}\right)$ when the contact line does not move; (c) withdrawing of liquid by pump (formation of the receding dynamic contact angle $\theta_{\mathrm{R}}$ ) 
Photo images of droplets were obtained by the shadow method. The latter was implemented by high-speed video camera FastVideo 500M (produced by FastVideo) 4 with macro lens Sigma $105 \mathrm{~mm}$ f/2.8G IF-ED AF-S 9 and source of plane-parallel light (Edmund Optics halogen light source with telecentric tube) 5. Goniometry methods were used to process experimentally obtained shadow images: Tangential 1 [31] - for dynamic and LB-ADSA [32] - for static contact angles. The static angles were averaged over the values of five measurements in different places of the surface; the dynamic experiments for obtaining dynamic contact angles and contact line speed were repeated five times in identical conditions. The random measurement error did not exceed $5 \%$.

Four textures were created on surfaces of AMG aluminum alloy (AlMg6 alloy: Al 91.2, Mg 6.8, Mn 0.8, Fe 0,4, Si 0.4, Zn 0.2, Ti 0.1, Cu 0.1 in wt \%) by abrasive discs with different grit size, one (polished) - by diamond paste. AMG aluminum alloy was chosen due to its widespread use in aviation, shipbuilding, automotive, and space technology [33]. The average grit sizes of abrasive discs and diamond paste are shown in Table 1.

Table 1 Abrasive grit (diamond paste and abrasive discs)

\begin{tabular}{|c|c|c|c|c|c|}
\hline $\begin{array}{c}\text { Marking of } \\
\text { material }\end{array}$ & $\begin{array}{c}\text { Paste ASM 1/0 } \\
\text { NVOM }\end{array}$ & $\begin{array}{c}\text { Disk } \\
\text { No 2500 }\end{array}$ & $\begin{array}{c}\text { Disk } \\
\text { No 1000 }\end{array}$ & $\begin{array}{c}\text { Disk } \\
\text { No 600 }\end{array}$ & $\begin{array}{c}\text { Disk } \\
\text { No 400 }\end{array}$ \\
\hline $\begin{array}{c}\text { Average size of } \\
\text { grit, } \mu \mathrm{m}\end{array}$ & less than 1 & 8.5 & 18.3 & 25.8 & 35.0 \\
\hline
\end{tabular}

Microstructure and microrelief of obtained surfaces were studied on Scanning electron microscope Hitachi-3000M and the Micromesure 3D Station profilometer (produced by STIL). Three-dimensional images of surfaces were processed in special software to obtain roughness parameters.

\section{Results and discussion}

\subsection{Effect of abrasive processing on texture of aluminum alloy surface}

Three-dimensional roughness parameters from ISO 25178-2:2012 [20] were chosen for a quantitative description of the surface texture (Fig. 3). They were obtained after processing profilograms. Amplitude parameters: Sa (the arithmetic mean height) characterizes the absolute values of surface deviation from the baseline in $\mu \mathrm{m}$; $\mathrm{Sz}$ (the maximum height of surface is defined as the average absolute value of the five highest peaks and the five deepest valleys) characterizes energy barriers [34] overcome by a moving contact line in $\mu \mathrm{m}$. Hybrid parameters: Sdq (the root mean square gradient of surface) is a degree of surface deviation from the molecularly smooth (dimensionless parameter) [20]:

$$
\mathrm{Sdq}=\sqrt{\frac{1}{A} \iint_{A}\left[\left(\frac{\partial z(x, y)}{\partial x}\right)^{2}+\left(\frac{\partial z(x, y)}{\partial y}\right)^{2}\right] d x d y,}
$$

and $\operatorname{Sdr}$ (developed interfacial area ratio of surface) is an increment of surface area in percentages [20]:

$$
\operatorname{Sdr}=\frac{1}{A}\left[\iint_{A}\left(\sqrt{\left[1+\left(\frac{\partial z(x, y)}{\partial x}\right)^{2}+\left(\frac{\partial z(x, y)}{\partial y}\right)^{2}\right]}-1\right) d x d y\right]
$$




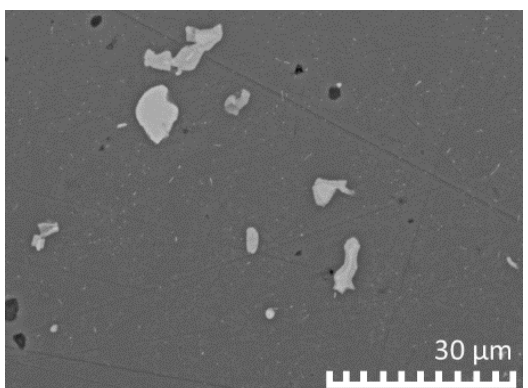

(a)

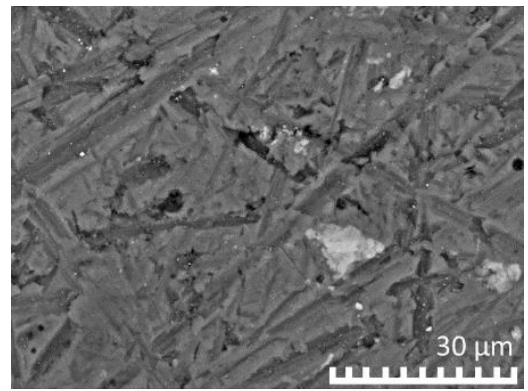

(b)

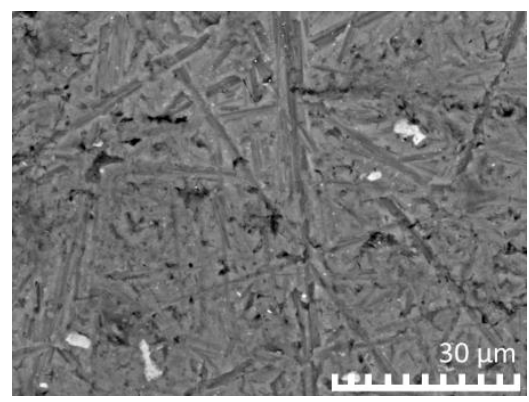

(c)

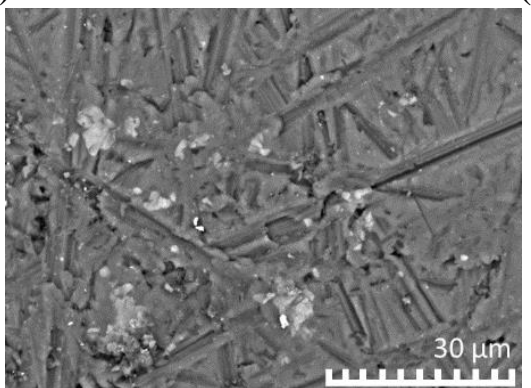

(d)

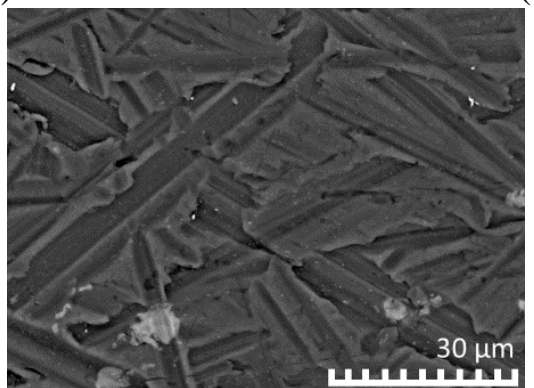

(e)

Fig. 3 Photo images of surfaces x2000: (a) polished; processed by abrasive discs with an average grit size, $\mu \mathrm{m}$ : (b) 8.5 ; (c) 18.3 ; (d) 25.8 ; (e) 35.0

Figure 4 shows the dependencies of the roughness parameters on the average grit size.

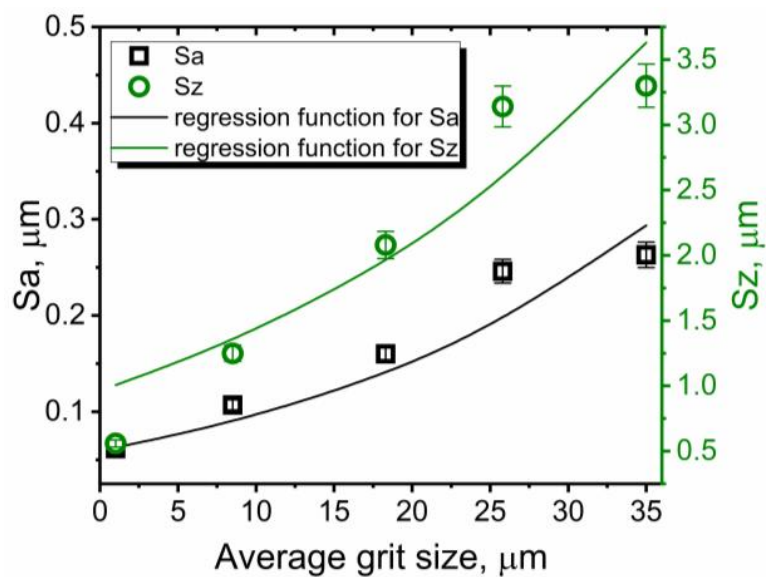

(a)

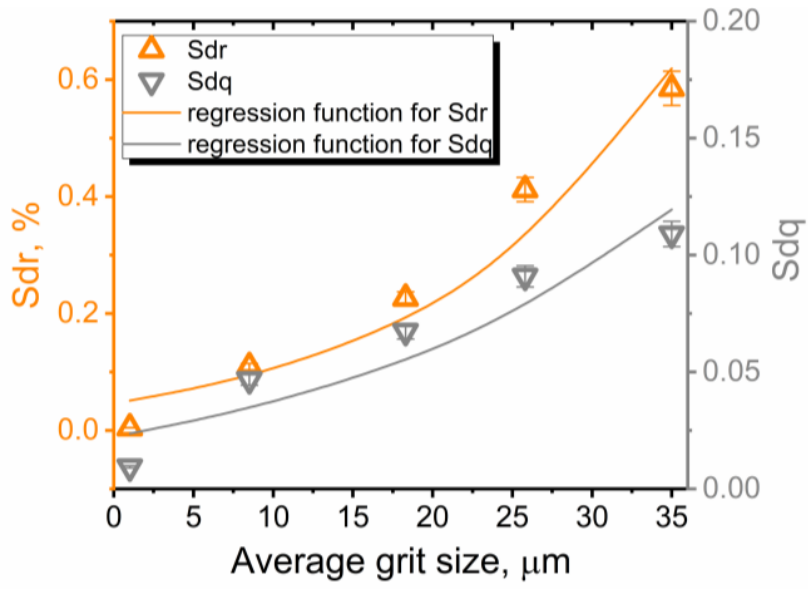

(b)

Fig. 4 Roughness parameters versus average grit size: (a) amplitude (Sa, the arithmetic mean height; Sz, the maximum height of surface); (b) hybrid (Sdq, the root mean square gradient of surface; Sdr, developed interfacial area ratio of surface)

It can be seen from Fig. 4 that an increase in the average grit size of the abrasive discs used for processing from 1 to $35 \mu \mathrm{m}$ led to an exponentially change (regression functions, Fig. 4) of the amplitude ( $\mathrm{Sa}, \mathrm{Sz})$ and hybrid (Sdq, Sdr) parameters. It should be noted that an increment of surface area after processing by the abrasive disc with the average grit size of $35 \mu \mathrm{m}$ was no more than $0.6 \%$ relatively molecularly smooth surface (Fig. 4 (b), regression function of Sdr). While the maximum height of irregularities increased 6 times, i.e. more than $3 \mu \mathrm{m}$ (Fig. 4 (a), 
regression function of $\mathrm{Sz}$ ), and the growth of $\mathrm{Sa}$ was $0.26 \mu \mathrm{m}$, which corresponds to four times increase.

It can be concluded that abrasive processing with varying of the average grit size in the range from 1 to $35 \mu \mathrm{m}$ creates a random texture consisting of multidirectional crossing micron-sized grooves on aluminum alloy surface. In such processing conditions, the surface area increases slightly, but local irregularities form on the surface texture, which are energy barriers that prevent the movement of a droplet on such surfaces.

\subsection{Wettability of abrasive processed aluminum alloy surfaces}

The effect of the texture (roughness) on wettability properties of created surfaces was analyzed. Figure 5 shows typical dependences of the static and dynamic contact angles on the amplitude $(\mathrm{Sa}, \mathrm{Sz})$ and hybrid $(\mathrm{Sdr}, \mathrm{Sq})$ roughness parameters. Presented in Fig. 5 dynamic angles were obtained for the flow rate of $5 \mu \mathrm{l} / \mathrm{s}$. The static and dynamic contact angles are smaller on textures characterized by large values of the roughness parameters (Fig. 5).

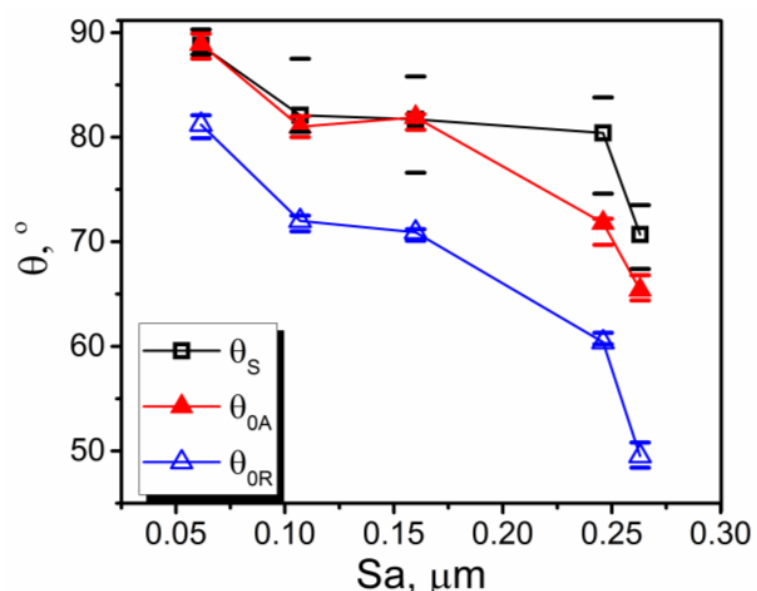

(a)

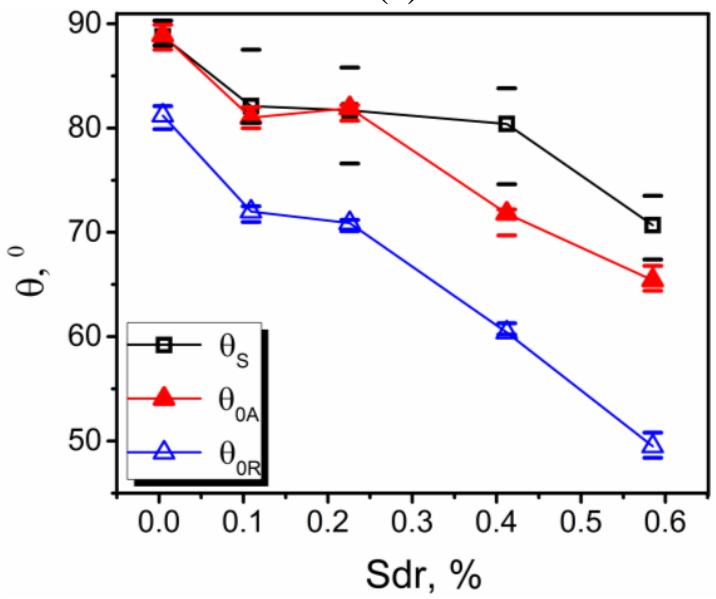

(c)

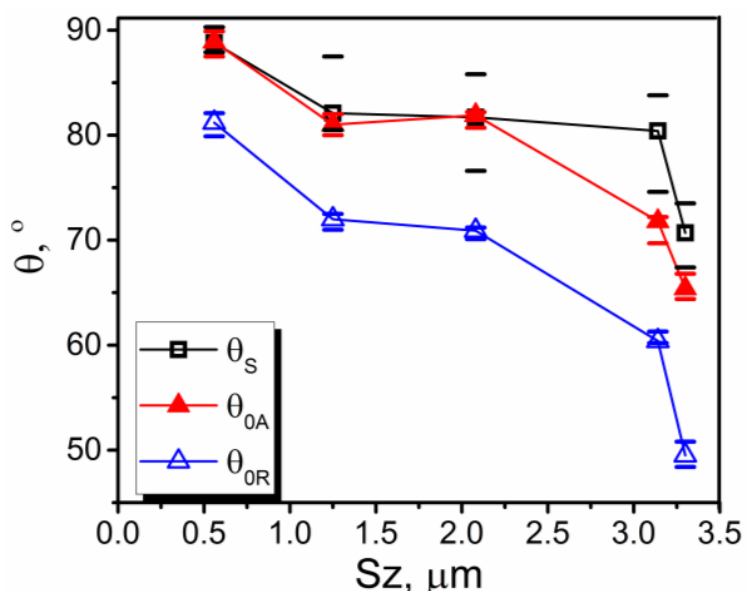

(b)

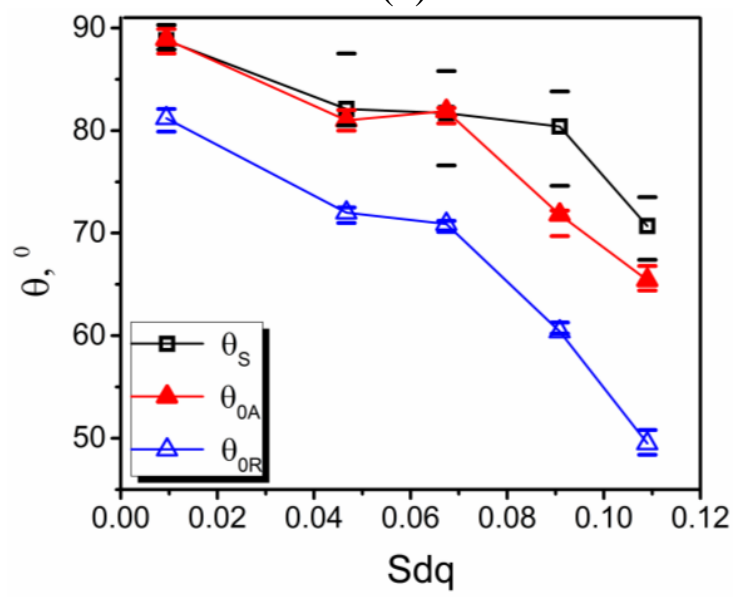

(d)

Fig. 5 Static and dynamic contact angles versus roughness parameters when the flow rate was equal to $5 \mu 1 / \mathrm{s}$ : (a), (b) amplitude; (c), (d) hybrid. The horizontal bars represent the scatter of the experimental data

The Wenzel-Derjaguin theory [35, 36] predicts that an increase in roughness of hydrophilic surface contributes to the enhancement of its hydrophilic properties and vice versa. According to the results of conducted experiments, an increase in roughness estimated by $\mathrm{Sa}, \mathrm{Sz}$ and $\mathrm{Sdr}$ 
parameters led to a decrease in the static contact angle, i.e. surfaces became more hydrophilic (Fig. 5). Since the polished aluminum alloy (close to molecularly smooth surface) shows hydrophilic properties, the results obtained are consistent with the Wenzel-Derjaguin theory. It should be noted that according to the Wenzel-Derjaguin equation $[35,36]$ for rough surface $\cos \theta_{r}=r \cdot \cos \theta$ the change in the contact angle is directly proportional to the roughness parameter $r$ (the ratio of the real area to its projection). In the case of presented results, the increment of the surface area characterized by Sdr was not more than $0.6 \%$ (Fig. 4 (b)). Consequently, the change in the contact angle is not directly proportional (Fig. 5) to the increment of the surface area. It can be assumed that the wettability properties after abrasive processing of the aluminum alloy surface, in addition to increasing the surface area, are significantly influenced by the geometric parameters of the texture elements (cavities and asperities) which are characterized by Sa and Sz parameters. However, according to the analysis of Figure 5 (a), the trivial dependence of the change in the contact angle on these parameters has not been established.

\subsection{Metastable state of a droplet on abrasive processed aluminum alloy surfaces}

The static contact angle is known to lie in the range from the advancing to receding dynamic contact angles measured on the same surface [37]: $\theta_{0 R} \leq \theta_{S} \leq \theta_{O A}$. It can be seem from Fig. 5 that the static contact angle on polished surface lies within this range. However, the static angle on surfaces processed by abrasive discs is greater than the advancing dynamic angle. Based on this, it is assumed that surface roughness is the cause of the metastable equilibrium of the "solidliquid-gas" system. According to the molecular-kinetic theory [8], the equilibrium state is established only when all kinetic energy of a droplet placed on molecularly smooth surface is dissipated. If the surface is rough, then the kinetic energy of the droplet after contacting the surface is dissipated before reaching the thermodynamically equilibrium state. The contact line pins on the irregularities, which leads to an increase in the static contact angle. In this case, the static contact angle is greater than the advancing dynamic angle. Thermodynamically equilibrium on a rough surface can be achieved by external influence, for example, vibration [37]. After its impact, the static contact angle decreases [37], the metastable state goes into the equilibrium; the static contact angle is in the range $\theta_{\mathrm{OR}} \leq \theta_{\mathrm{S}} \leq \theta_{\mathrm{OA}}$. It is worth noting that with an increase in the flow rate from 5 to $100 \mu \mathrm{l} / \mathrm{s}$ the deviation from the equilibrium state increases (the difference between the static and the advancing dynamic contact angle increases).

\subsection{Effect of liquid flow rate and texture on spreading dynamics}

Contact angle hysteresis $(\Delta \theta)$ was determined as the difference between the advancing and receding dynamic contact angles [6] when the contact line movement is equal to zero. The dependencies of the $\mathrm{CAH}$ on the roughness parameters are shown in Fig. 6. 


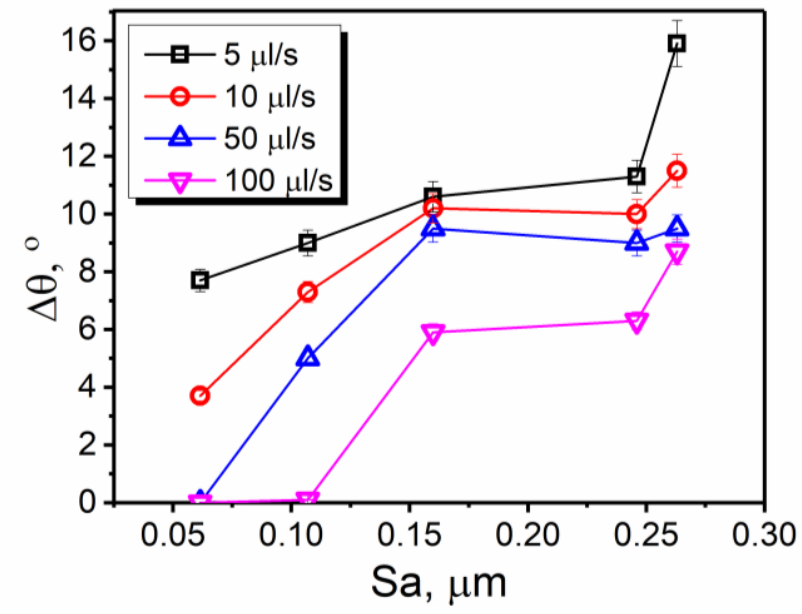

(a)

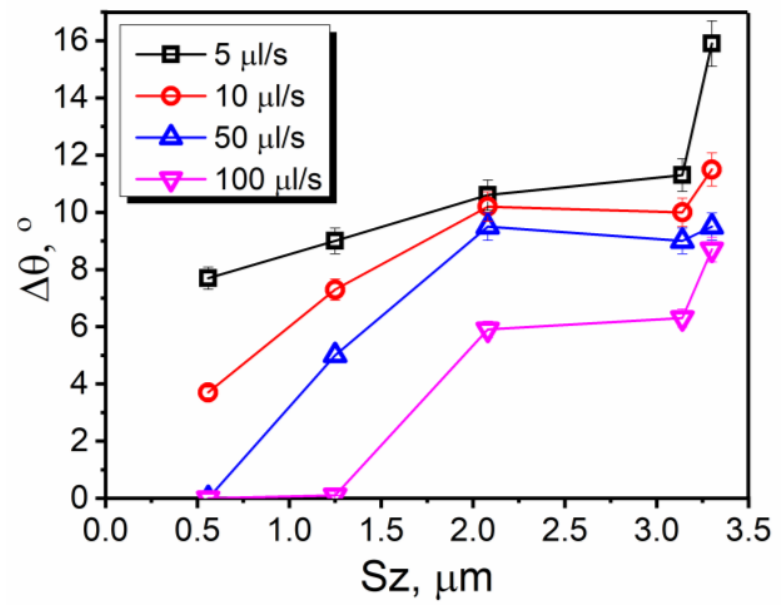

(b)

Fig. 6 Contact angle hysteresis versus amplitude roughness parameters at different liquid flow rates: (a) $\mathrm{Sa}$; (b) $\mathrm{Sz}$

When the flow rate is $100 \mu \mathrm{l} / \mathrm{s}$, the CAH is less than $1^{\circ}$ on surfaces processed by abrasive discs with an average grit size of less than $8.5 \mu \mathrm{m}$. This is no more than the measurement error. The dependence of $\Delta \theta$ on the roughness parameters (Fig. 6) in the range from 18.3 to $35 \mu \mathrm{m}$ increases. Reducing the liquid flow rate from 100 to $5 \mu \mathrm{l} / \mathrm{s}$ increases the $\mathrm{CAH}$. This can be explained by the fact that after abrasive processing, chaotically located grooves are formed on the surface in different directions (Fig. 3). They are energy barriers that impede the movement of a droplet. The quantitative characteristics of these irregularities are $\mathrm{Sa}$ and $\mathrm{Sz}$ parameters. The first of them characterizes the average size of irregularities along the horizon in the direction the contact line movement, the second is the maximum size of ten irregularities in the vertical. It is worth noting the similar nature of the dependences of $\mathrm{Sa}$ and $\mathrm{Sz}$ on the average grit size (Fig. 4). When a droplet spreads over the surface, the contact line pins over irregularities (energy barriers preventing its movement). If the kinetic energy of droplet depending on the flow rate is sufficient to overcome the energy barriers, then liquid spreads smoothly without pinning, and the CAH in this case is minimal. The spreading of a droplet with flow rate of $100 \mu \mathrm{l} / \mathrm{s}$ over surfaces with Sa and $\mathrm{Sz}$ no more than 0.11 and $1.25 \mu \mathrm{m}$, respectively, correspond to this case in Fig 6. At the same flow rate an increase in the hysteresis to $5.9^{\circ}$ was recorded due to roughness on surfaces with $\mathrm{Sa}>0.16 \mu \mathrm{m}$ and $\mathrm{Sz}>2.08 \mu \mathrm{m}$. In this case, the kinetic energy is insufficient to overcome irregularities and the CL pins. With increasing the flow rate from 100 to $5 \mu \mathrm{l} / \mathrm{s}$ the kinetic energy decreases, and it is impossible to overcome the irregularities on surfaces with Sa and $\mathrm{Sz}$ less than 0.11 and $1.25 \mu \mathrm{m}$, that causes contact line pinning and an increase in the hysteresis (Fig. 6), which does not contradict conclusions in [38]. Thus, it can be concluded that the contact angle hysteresis depends on the geometrical dimensions of the irregularities on the surface and the kinetic energy of the droplet.

Figure 7 presents typical time dependencies of the contact line speed over surfaces with random roughness for the case when the flow rate was equal to $5 \mu \mathrm{l}$. For convenience, the positive values of the contact line speed correspond to the advancing contact line (towards the gas phase when the pump dispensed water), the negative ones - to the receding contact line (towards the liquid phase when the pump withdrew water). 


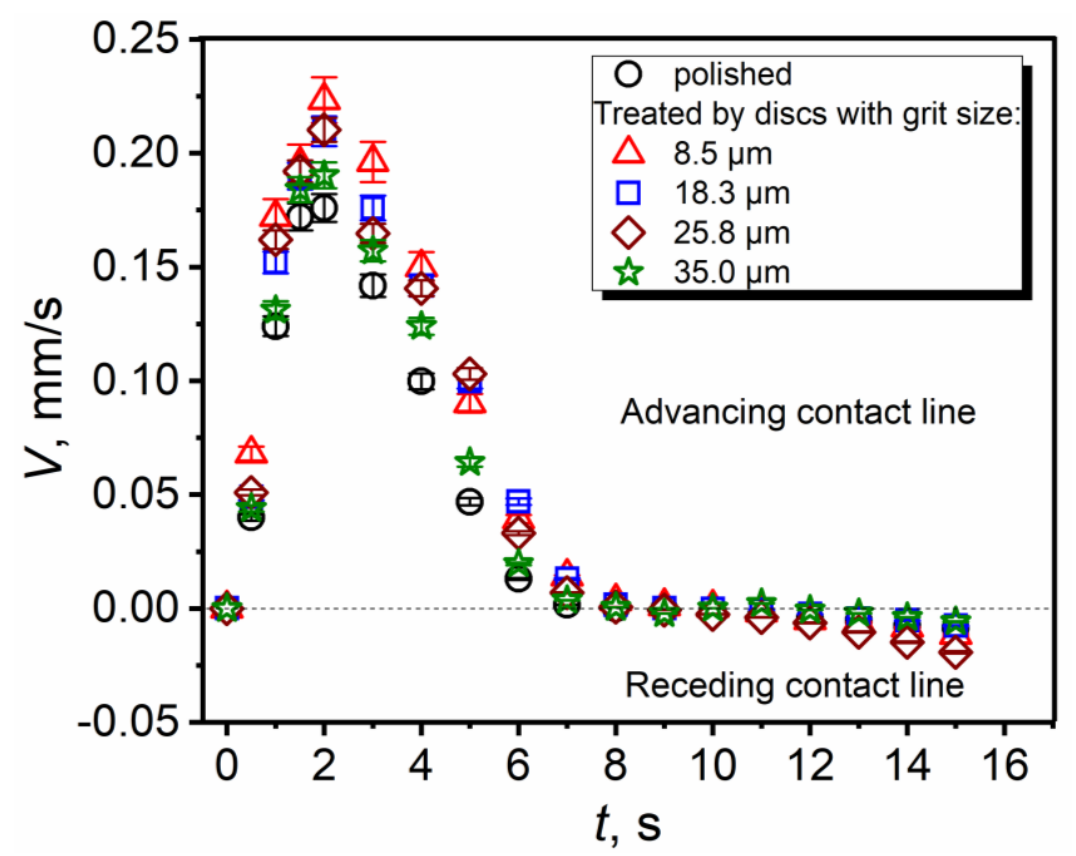

Fig. 7 Time dependencies of the contact line speed when the flow rate is $5 \mu 1 / \mathrm{s}$

Curves corresponding to surfaces processed by abrasive discs with different average grit size have the same nature; therefore, the surface roughness did not have a significant effect on the contact line speed. The dominant factor affecting the contact line speed is the flow rate (with its growth the CL speed increases).

\subsection{Interpretation of dynamic contact angle data by molecular-kinetic model}

Obtained experimental dependencies of the dynamic contact angle on the contact line speed were interpreted by the classic (2) and linear (3) molecular-kinetic equations [8] (Fig. 8). The hydrodynamic theory [11] was not an appropriate model and was not used as in [39] due to the effect of pinning and the presence of the partial slip-condition along the rough surfaces.

The list squares method was used to compare the experimental dependences of DCAs on the CL speed with calculated according to eq. (2) and (3). Values of independent parameters $\xi, k^{0}$, $\lambda$ in eq. (2) and (3) were taken according to [12, 28, 40-44] in a first approximation, in subsequent iterations their values were adjusted to minimize the sum of squares of the deviations between the calculated dynamic contact angles and the experimental data. The goodness-of-fit coefficient $\mathrm{R}^{2}$ was used to evaluate converging between experimental and theoretical data, and parameters $\xi, k^{0}, \lambda$ were calculated. The equilibrium contact angle $\theta$ in eq. (2) and (3) was set as independent parameter. Advancing and receding data were fitted by eq. (2) and (3) separately in order to exclude the contact angle hysteresis. A large deviation of the experimental data from the theoretical dependences (2) and (3) was found in [28] when the contact angle hysteresis was $5 \circ$. Obtained CAHs on surfaces with random roughness exceed this value. Therefore, it is possible to assume that the deviations will be even more significant in this case. It follows that the values of equilibrium contact angles in the MKT eq. (2) and (3) correspond to the advancing $\theta_{0 \mathrm{~A}}$ and receding $\theta_{0 \mathrm{R}}$ dynamic contact angles when the CL pins. 


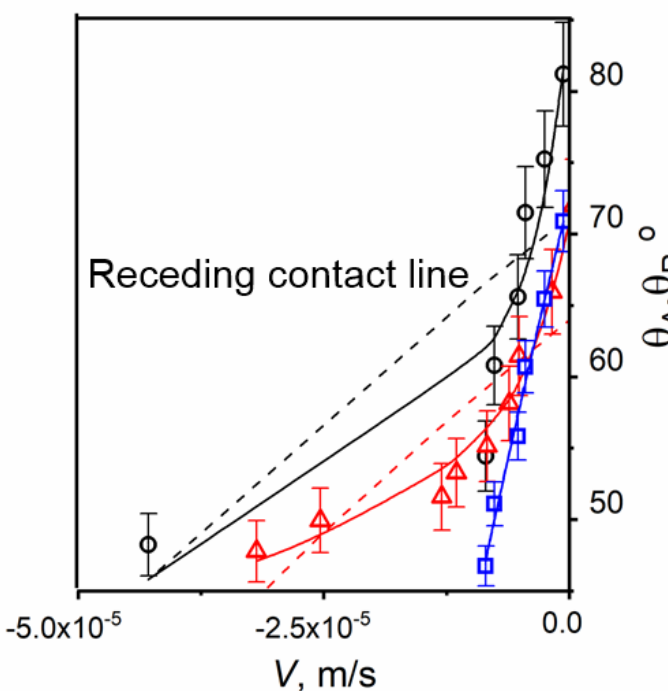

(a)

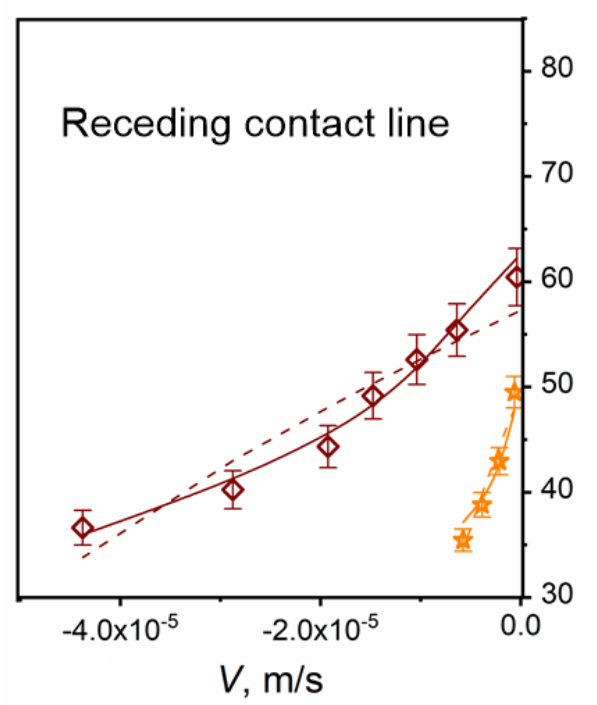

(c)

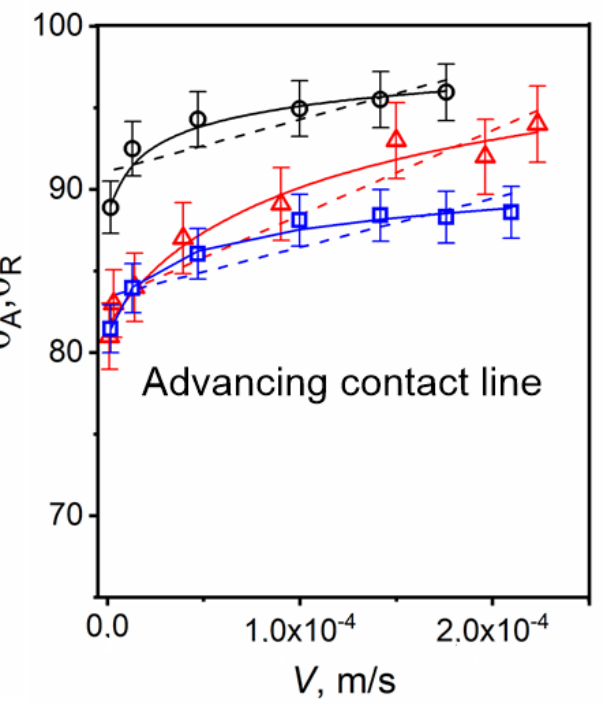

(b)

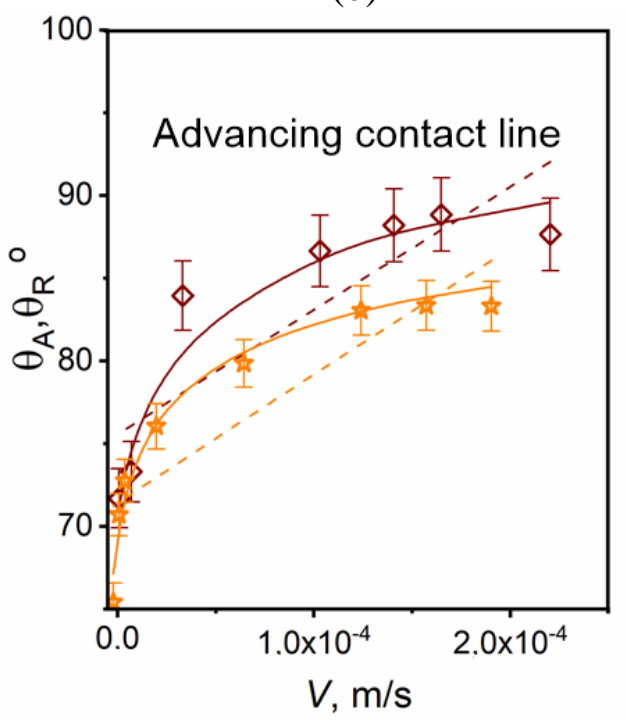

(d)

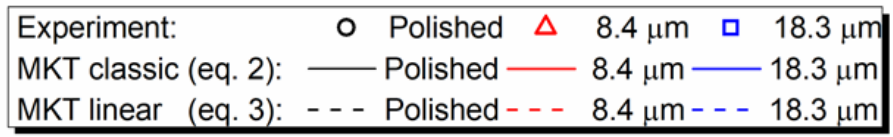

\begin{tabular}{llr}
\hline Experiment: & $\diamond 25.8 \mu \mathrm{m}$ \& $35.0 \mu \mathrm{m}$ \\
MKT classic (eq. 2): & $-25.8 \mu \mathrm{m}-35.0 \mu \mathrm{m}$ \\
MKT linear (eq. 3): & $---25.8 \mu \mathrm{m}---35.0 \mu \mathrm{m}$ \\
\hline
\end{tabular}

Fig. 8 Dynamic contact angles versus contact line speed for prepared surfaces: (a), (c) receding movement; (b), (d) advancing movement. Symbols correspond to experimental data, lines MKT [8]

Calculated parameters obtained by fitting of the experimental data $(5 \mu 1 / \mathrm{s})$ and theoretical dependencies (2) and (3) are presented in Table 2. It can be concluded that when using the classic MKT eq. (2), the deviation is smaller. An average displacement distance of molecules $\lambda$ varies within $0.9 \div 2.1 \mathrm{~nm}$, which is greater than the size of water molecules $0.28 \mathrm{~nm}$. Obtained range is consistent with calculations presented in [12, 28, 40-44]. An equilibrium frequency $k^{0}$ of molecular displacements changes within $k^{0}=141.2 \div 2957.4 \mathrm{~Hz}$ and differs from obtained in [28] for advancing and receding movement of liquid over polished aluminum-magnesium alloy. It is connected with the higher contact line speeds due to flow rates $(50-110 \mu \mathrm{l} / \mathrm{s})$ which are larger than in the present study. This led to an increase in the equilibrium frequency varying 
within $10^{3}-10^{4} \mathrm{~Hz}$ in [28]. Such a wide range of variation $k^{0}=141.2 \div 2957.4 \mathrm{~Hz}$ can be explained by the chaotic arrangement of the roughness elements.

Table 2 Parameters obtained by fitting of the experimental data and theoretical dependencies

\begin{tabular}{|c|c|c|c|c|c|c|c|c|}
\hline \multirow{2}{*}{$\begin{array}{c}\text { Surface } \\
\text { processed by } \\
\text { paste/discs with } \\
\text { average grit } \\
\text { size }\end{array}$} & \multirow{2}{*}{$\begin{array}{c}\text { Experimentally } \\
\text { obtained } \\
\text { angles } \\
\theta_{\mathrm{OA}} / \theta_{\mathrm{OR}},{ }^{\circ}\end{array}$} & \multicolumn{4}{|c|}{ Classic MKT (eq. 2) } & \multicolumn{3}{|c|}{ Linear MKT (eq. 3) } \\
\hline & & $\theta, \circ$ & $k^{0}, \mathrm{~Hz}$ & $\lambda, \mathrm{nm}$ & $\mathrm{R}^{2}$ & $\theta, \circ$ & $\xi, \mathrm{Pa} \cdot \mathrm{s}$ & $\mathrm{R}^{2}$ \\
\hline \multicolumn{9}{|c|}{ Advancing contact line $\theta_{\mathrm{A}}(V)$} \\
\hline Polished & 88.9 & 86.4 & 141.2 & 2.1 & 0.99 & 91.1 & 40.0 & 0.63 \\
\hline 8.4 & 81.0 & 81.6 & 331.8 & 1.9 & 0.91 & 83.2 & 65.1 & 0.80 \\
\hline 18.3 & 81.5 & 80.8 & 1121.5 & 1.9 & 0.99 & 83.5 & 37.5 & 0.78 \\
\hline 25.8 & 71.7 & 70.7 & 2216.4 & 1.2 & 0.96 & 75.6 & 92.8 & 0.70 \\
\hline 35.0 & 65.4 & 69.1 & 2957.4 & 1.4 & 0.99 & 71.3 & 94.5 & 0.86 \\
\hline \multicolumn{9}{|c|}{ Receding contact line $\theta_{\mathrm{R}}(V)$} \\
\hline Polished & 81.2 & 83.7 & 542.1 & 0.9 & 0.88 & 71.2 & 626.8 & 0.58 \\
\hline 8.4 & 72.0 & 70.9 & 740.1 & 1.1 & 0.98 & 64.0 & 619.5 & 0.71 \\
\hline 18.3 & 70.9 & 72.7 & 521.2 & 1.0 & 0.83 & 72.4 & 3189.4 & 0.99 \\
\hline 25.8 & 60.4 & 63.4 & 771.3 & 1.2 & 0.94 & 57.7 & 486.5 & 0.87 \\
\hline 35.0 & 49.5 & 53.5 & 401.1 & 1.1 & 0.99 & 49.4 & 2164.9 & 0.94 \\
\hline
\end{tabular}

Experimental data is poorly fitted by the linear MKT eq. (3) (Fig. 8). It is connected with nonlinear dependence of the advancing/receding dynamic contact angle on the contact line speed. An exception is the fitting of the DCA data on the surface processed by the disc with the average grit size of $18.3 \mu \mathrm{m}$. In this case, due to the linear form of $\theta_{\mathrm{R}}=f(V)$, the eq. (3) better fits the experimental data compared to the eq. (2). For the classic MKT equation (2) $\mathrm{R}^{2}$ is 0.83 , for linear eq. (3) $\mathrm{R}^{2}$ is 0.99 . The friction coefficient of the contact line $\xi$ in eq. (3) varies within $37.5 \div 92.8 \mathrm{~Pa} \cdot \mathrm{s}$ for the advancing contact line and $486.5 \div 3189.4 \mathrm{~Pa} \cdot \mathrm{s}$ for the receding CL. When water droplet spread on PET [41], it was equal to $0.01 \mathrm{~Pa} \cdot \mathrm{s}$. However, for liquids with higher viscosity, for example, silicone oil on glass [45], $\xi$ reached $3580 \mathrm{~Pa} \cdot \mathrm{s}$. The values of the friction coefficients can be considered as physically reasonable since surfaces with higher roughness compared to PET were used in these studies, that is, sufficiently large friction of the liquid took place, due to which pinning of the contact line occurs.

\section{Conclusion}

A quantitative assessment of the textures formed on aluminum-magnesium alloy by abrasive discs with different average grit size was conducted. Two amplitude and hybrid threedimensional parameters were selected as the roughness characteristics. These parameters were found to increase exponentially with increasing the grit size from 1 to $35 \mu \mathrm{m}$. Aluminummagnesium alloy surface area increased slightly after abrasive processing with an average grit size of $35 \mu \mathrm{m}$, and the static contact angle decreases from $88.8^{\circ}$ to $70.7^{\circ}$. Since the molecularly smooth surface of AlMg6 alloy demonstrates hydrophilic properties, the experimental contact angles measured after abrasive processing are in qualitative agreement with the WenzelDerjaguin theory $[35,36]$. Nevertheless, a decrease in the experimental static contact angle does not agree quantitatively with the results of calculations by the Wenzel-Derjaguin equation [35, 36]. As far as the elemental composition of surfaces does not change after abrasive processing as 
opposed to nanosecond laser texturing [46], the change in wettability is supposed to be dependent not only on an increase in the surface area due to roughness but on the geometric shape of texture elements, cavities, and asperities, characterized by three-dimensional roughness parameters [20]. Experimentally proved usage of correction coefficients taking into account the geometry of texture elements in the Wenzel-Derjaguin equation [35, 36] requires to conduct similar experimental studies on metals demonstrating both hydrophobic and hydrophilic properties when their surface is molecularly smooth.

A droplet of distilled water is found to be in the metastable state on AlMg6 alloy processed by abrasive discs. It is characterized by large contact angle hysteresis and inequality $\theta_{\mathrm{OR}} \leq \theta_{\mathrm{OA}} \leq \theta_{S}$. The deviation from the equilibrium state, in addition to the texture, is influenced by the liquid flow rate. The last mention also influences the contact angle hysteresis. To shift the metastable state of a droplet on the steady state is possible by applying vibration [37]. The steady state of droplets on the surface is important for technologies where drop dispensing is implemented, for example, irrigation cooling systems, and microfluidic devices.

Obtained experimental dependencies of the dynamic contact angle on the contact line speed were fitted by the molecular-kinetic theory (linear and classic equations). The best fit was obtained with classic equation and experimental data.

\section{Acknowledgment}

The reported study was supported by RFBR, research project No. 18-38-00315 mol_a. The optical shadow system was elaborated at Tomsk Polytechnic University within the framework of Tomsk Polytechnic University Competitiveness Enhancement Program grant.

\section{References}

1. B. Derby, Annu. Rev. Mater. Res. 40, 395 (2010)

2. A.A. Darhuber, S.M. Troian, Annu. Rev. Fluid. Mech. 37, 425 (2005)

3. D. Anton, Surface-fluorinated coatings, Adv. Mater. 10(15), 1197 (1998)

4. A. Borruto, G. Crivellone, F. Marani, Wear 222(1), 57 (1998)

5. T. Young, Philos. Trans. R. Soc. Lond. 95, 65 (1805)

6. $\quad$ E.B.V. Dussan, Annu. Rev. Fluid Mech. 11, 371 (1979)

7. J.-H. Kim, H.P. Kavehpour, J.P. Rothstein, Phys. Fluids 27, 032107 (2015)

8. T.D. Blake, J.M. Haynes, J. Colloid Interface Sci. 30, 421 (1969)

9. E. Ruckenstein, C.S. Dunn, J. Colloid Interface Sci. 59, 135 (1977)

10. R.G. Cox, J. Fluid Mech. 168, 169 (1986)

11. O.V. Voinov, J. Fluid Dyn. 11, 714 (1976)

12. R.A. Hayes, J. Ralston, J. Colloid Interface Sci. 159, 429 (1993)

13. S.-Y. Chen, Y. Kaufman, A.M. Schrader, D. Seo, D.W. Lee, S.H. Page, P.H. Koenig, S. Isaacs, Y. Gizaw, J.N. Israelachvili, Langmuir 33, 10041 (2017)

14. J. Long, P. Fan, D. Gong, D. Jiang, H. Zhang, L. Li, M. Zhong, ACS Appl. Mater. Interfaces 7, 9858 (2015)

15. A.L. Dubov, A. Mourran, M. Möller, O.I. Vinogradova, J. Chem. Phys. 141, 074710 (2014)

16. S.Y. Misyura, Chem. Eng. Res. Des. 129, 306 (2018)

17. S.Y. Misyura, Int. Commun. Heat Mass Transf. 96, 7 (2018)

18. K.J. Kubiak, M.C. Wilson, T.G. Mathia, S. Carras, Scanning 33, 370 (2011)

19. K.J. Kubiak, M.C.T. Wilson, T.G. Mathia, Ph. Carval, Wear 271, 523 (2011) 
20. ISO 25178-2:2012. Geometrical product specifications (GPS) - Surface texture: Areal Part 2: Terms, definitions and surface texture parameters.

21. V. Hejazi, A.D. Moghadam, P. Rohatgi, M. Nosonovsky, Langmuir 30, 9423 (2014)

22. H. Gu, C. Wang, S. Gong, Y. Mei, H. Li, W. Ma, Surf. Coat. Tech. 292, 72 (2016)

23. E.A. Papp, C. Csiha, Surfaces and Interfaces 8, 54 (2017)

24. R.N. Wenzel, J. Phys. Chem. 53, 1466 (1949)

25. A.B.D. Cassie, S. Baxter, Nature 155, 21 (1945)

26. B. Zahiri, P.K. Sow, C.H. Kung, W. Mérida, J. Colloid Interface Sci. 501, 34 (2017)

27. S.Giljean, M. Bigerelle, K. Anselme, H. Haidara, Appl. Surf. Sci. 257(22), 9631 (2011)

28. E.G. Orlova, D.V. Feoktistov, G.V. Kuznetsov, K.O. Ponomarev, Eur. J. Mech. B/Fluids 68, $118(2018)$

29. G.V. Kuznetsov, D.V. Feoktistov, E.G. Orlova, K. Batishcheva, S.S. Ilenok, Appl. Surf. Sci. 469, 974 (2019)

30. Zaitsev, D.V., Kirichenko, D.P., Ajaev, V.S., Kabov, O.A., Phys. Rev. Lett. 119, 094503 (2017)

31. A. Bateni, S.S. Susnar, A. Amirfazli, A.W. Neumann, Colloids Surf. A Physicochem. Eng. Asp. 219, 215 (2003)

32. F. Bashforth, J.C. Adams, An attempt to test the theories of capillary action by comparing the theoretical and measured forms of drops of fluid (Cambridge Univ. Press, London, 1883) p. 162.

33. A. D. Modestov, K. A. Emelyanenko, A. M. Emelyanenko, A. G. Domantovsky, L. B. Boinovich, Russian Chemical Bulletin 65, 2607 (2016)

34. P.G. de Gennes, Wetting: Statics and Dynamics, Rev. Mod. Phys. 57, 827 (1985)

35. B.V. Derjaguin, Dokl. Akas. Nauk SSSR 51, 357 (1946)

36. R.N. Wenzel, Resistance of solid surfaces to wetting by water, Ind. Eng. Chem. 28, 988 (1936)

37. M. Kanungo, S. Mettu, K.-Y. Law, S. Daniel, Langmuir 30, 7358 (2014)

38. R.E. Johnson, R.H. Dettre, in: Wettability, ed. By J.C. Berg (Marcel Dekker, New York, 1993) pp. 2-71.

39. A. Mohammad Karim, J.P. Rothstein, H. P. Kavehpour, J. Colloid Interface Sci. 513, 658 (2018)

40. R. Sedev, Adv. Colloid Interface Sci. 222, 661 (2015)

41. T.D. Blake, in: Wettability, ed. By J.C. Berg (Marcel Dekker, New York, 1993) pp. 251309.

42. S.R. Ranabothu, C. Karnezis, L.L. Dai, J. Colloid Interface Sci. 288, 213 (2005)

43. R. Fetzer, J. Ralston, J. Phys. Chem. C 113, 8888 (2009)

44. R.A. Hayes, J. Ralston, Langmuir 10, 340 (1994)

45. R.L. Hoffman, J. Colloid Interface Sci. 50, 228 (1975)

46. L.B. Boinovich, E.B. Modin, A.R. Sayfutdinova, K.A. Emelyanenko, A.L. Vasiliev, A.M. Emelyanenko, ACS Nano 11(10), 10113 (2017) 